\title{
Platinum composite nanowires for ultrasensitive mass detection
}

Tingting Hao, Tiehan H. Shen, Wuxia Li, Chenzhi Song, Zhi Xu, Aizi Jin, Ling Jin, Junjie Li, Xuedong Bai, and Changzhi Gu

Citation: Appl. Phys. Lett. 110, 143102 (2017);

View online: https://doi.org/10.1063/1.4979645

View Table of Contents: http://aip.scitation.org/toc/apl/110/14

Published by the American Institute of Physics

\section{Articles you may be interested in}

High temperature efficient, stable Si wafer-based selective solar absorbers

Applied Physics Letters 110, 141101 (2017); 10.1063/1.4979510

Broadband light absorber based on porous alumina structure covered with ultrathin iridium film Applied Physics Letters 110, 141103 (2017); 10.1063/1.4979581

Optimal design of nanoporous materials for electrochemical devices

Applied Physics Letters 110, 143103 (2017); 10.1063/1.4979466

Hotspot cooling with jumping-drop vapor chambers

Applied Physics Letters 110, 141601 (2017); 10.1063/1.4979477

Single photon extraction from self-assembled quantum dots via stable fiber array coupling

Applied Physics Letters 110, 142104 (2017); 10.1063/1.4979827

Ultra-sensitive NEMS magnetoelectric sensor for picotesla DC magnetic field detection

Applied Physics Letters 110, 143510 (2017); 10.1063/1.4979694

\section{Scilight}

Sharp, quick summaries illuminating the latest physics research

\section{Sign up for FREE!}

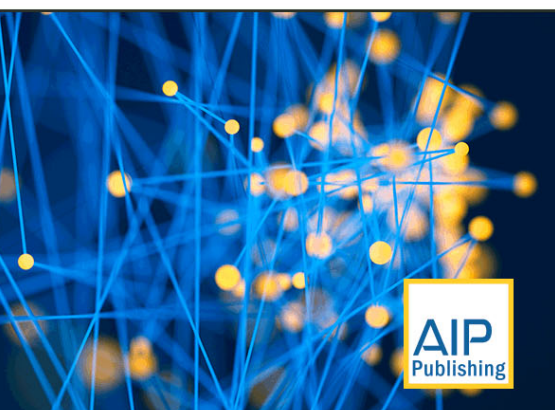




\title{
Platinum composite nanowires for ultrasensitive mass detection
}

\author{
Tingting Hao, ${ }^{1,2, a)}$ Tiehan H. Shen, ${ }^{3}$ Wuxia Li, ${ }^{1, b)}$ Chenzhi Song, ${ }^{1}$ Zhi Xu, ${ }^{1}$ Aizi Jin, ${ }^{1}$ \\ Ling Jin, ${ }^{1}$ Junjie Li, ${ }^{1}$ Xuedong Bai, ${ }^{1,4}$ and Changzhi Gu ${ }^{1,4,2, b)}$ \\ ${ }^{1}$ Beijing National Lab of Condensed Matter Physics, Institute of Physics, Chinese Academy of Sciences, \\ Beijing 100190, China \\ ${ }^{2}$ School of Physical Sciences, CAS Key Laboratory of Vacuum Physics, University of Chinese Academy of \\ Sciences, Beijing 100190, China \\ ${ }^{3}$ Joule Physics Laboratory, University of Salford, Manchester M5 4WT, United Kingdom \\ ${ }^{4}$ Collaborative Innovation Center of Quantum Matter, Beijing 100190, China
}

(Received 20 December 2016; accepted 19 March 2017; published online 3 April 2017)

\begin{abstract}
Platinum (Pt) composite nanowires were grown on the tip of tungsten (W) microprobes by focusedelectron-beam induced chemical vapor deposition (FEB-CVD). An electrical field was used to drive a transversal mechanical vibration of the nanowires. Such nanowire vibrations were found to display the first and second harmonic resonances with frequencies in the range of tens of $\mathrm{MHz}$. The Young's modulus of the nanowires was estimated to be in the range of $(1.4 \pm 0.1) \times 10^{2} \mathrm{GPa}$ to $(4.7 \pm 0.2) \times 10^{2} \mathrm{GPa}$, dependent on the wire size. A mass responsivity of $2.1 \times 10^{21} \mathrm{~Hz} / \mathrm{kg}$ was demonstrated with the minimum detectable mass of about 0.4 attogram. Our results indicated the potentials of FEB-CVD for the fabrication of nano-balances on any surface for ultra-sensitive mechanical applications. Published by AIP Publishing. [http://dx.doi.org/10.1063/1.4979645]
\end{abstract}

Nanoelectromechanical systems (NEMSs) are characterized by high mass sensitivity, high resonance frequency, and high quality factor, which can be utilized for the conversion of electrical signals into mechanical motions. ${ }^{1}$ The vibrational frequencies of such NEMSs are highly sensitive over a wide mass range with minuscule active masses. As such, they are favorable candidates for the detection of a single molecule, which would require a mass sensitivity in the range of zeptogram to attogram $\left(10^{-21}-10^{-18} \mathrm{~g}\right),{ }^{2}$ NEMSs have a strong potential in a wide range of applications, such as in force sensing ${ }^{3}$ and mass resonator, ${ }^{2,4}$ as well as in the study of quantum vibration ${ }^{5}$ and other fundamental physical phenomena. ${ }^{6}$

To achieve ultra-high mass sensitivity, various low dimensional structures ${ }^{2,7-12}$ have been subjected to detailed investigations. Among them, freestanding nanowires have attracted much attention since they would naturally work in the single-clamped mode with minimal energy loss. ${ }^{9}$ The focused-ion-beam (FIB) and the focused-electron-beam (FEB) induced chemical-vapor-deposition (CVD), which can fabricate freestanding nanowires at any given location, have been recognized as the most favorable approach in such device prototyping. ${ }^{10}$ Of these two techniques, the FEBCVD process usually has a lower growth rate but a higher carbon content due to the incomplete decomposition of the metal-organic precursor molecules, but it has the advantage of growing nanowires with a smaller diameter.

With the aim of obtaining nanowires of a smooth surface morphology, a small diameter, and a high Young's modulus, we chose FEB-CVD as the growth method for the present work. It is known that the material characteristics of a nanostructure, such as the Young's modulus, E, may differ from

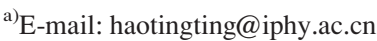

${ }^{b)}$ Authors to whom correspondence should be addressed. E-addresses:

liwuxia@aphy.iphy.ac.cn and czgu@aphy.iphy.ac.cn
}

those of the bulk material when different growth methods and parameters are used. ${ }^{11,13}$ Therefore, a practical method was used to measure $E$ non-destructively for nanowires of different sizes, the details of which are to be described later in this paper.

We report the observation of the first and the second harmonic resonances at several $\mathrm{MHz}$ in vibrations transversal to the nanowires. A detection of a change in mass with a sensitivity of $2.1 \times 10^{21} \mathrm{~Hz} / \mathrm{kg}$ and a minimum detectable mass of about 0.4 attogram is demonstrated. Our results suggest that the FEB-CVD technique has a strong potential for the integration of freestanding nanowires into appropriate devices for high-performance electro-mechanical applications.

The freestanding platinum $(\mathrm{Pt})$ composite nanowires were grown with a dual beam FIB-SEM (Helios 600i, FEI) system using an $86 \mathrm{pA}$ electron beam current with an accelerating voltage of $5.0 \mathrm{kV}$. The CVD precursor was $\left(\mathrm{CH}_{3}\right)_{3} \mathrm{Pt}\left(\mathrm{CpCH}_{3}\right)$. The mechanical vibrations of the nanowires were recorded by using a real-time JEOL 2010 FEG transmission electron microscope (TEM), and the images were taken using a screen capture software (e.g., Wink) installed in the system. Two in-house built sample holders installed in the TEM were used to conduct the experiments for the determination of the Young's modulus and the density of the freestanding nanowires and to electrically drive the mechanical vibration of the nanowires.

The mechanical measurement was realized by the freestanding Pt composite nanowire deposited on the tungsten (W) tip and actuated with a piezoelectric actuator to approach the stationary AFM cantilever. The schematic diagram in Fig. 1(a) illustrates the in situ measurement on the mechanical deformation of the nanowires. This enables the determination of the critical force, $F_{\mathrm{cr}}$, which in turn leads to the determination of the Young's modulus, $E$, based on the Euler buckling model. ${ }^{14,15}$ The solid-lined schematic drawing shows that the moment of the nanowire, being moved by a piezoelectrically driven stage, is just in touch with the 
(a)
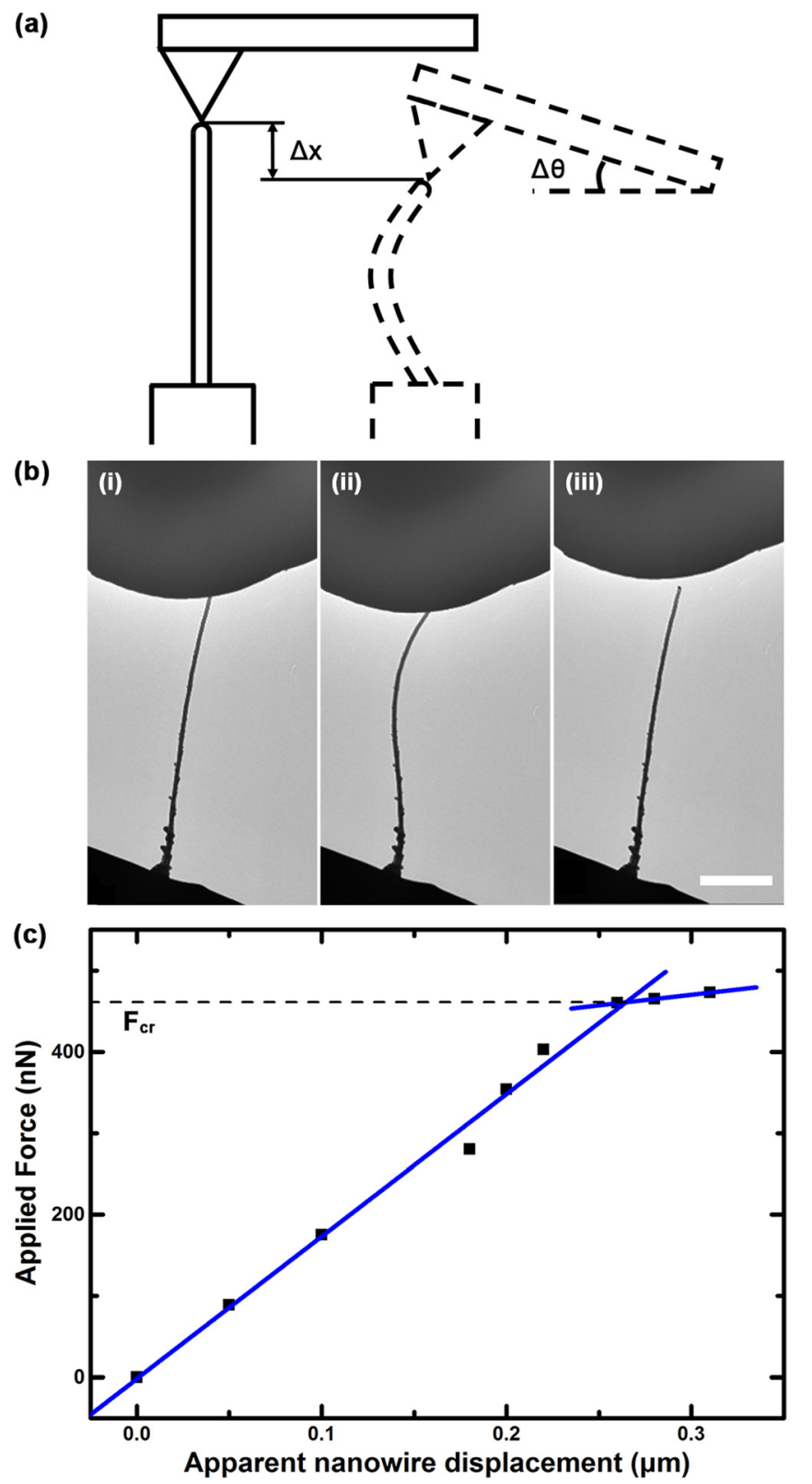

FIG. 1. The determination of the Euler buckling critical force (Fcr): (a) A schematic diagram showing an AFM tip at the moment just in contact with a nanowire (solid-lined) and when the wire is buckled (dash-lined). $\Delta \mathrm{x}$ indicates that the distance of the nanowire is compressed, and $\Delta \theta$ symbolically indicates the deflection of the AFM cantilever. (b) TEM images of a nanowire at different stages of compression: (i) just touching the AFM tip, (ii) the wire is buckled under the load, and (iii) the wire restores to its original shape when the load is removed, indicating the elastic deformation of the buckling. (c) Corresponding curve of the applied force versus the apparent nanowire displacement $(\Delta \mathrm{x})$ when the nanowire is buckled. The scale bar is $1 \mu \mathrm{m}$.

AFM cantilever. The dash-lined drawing shows that the wire is buckled under the load applied by the cantilever. As the nanowire is pushed towards the cantilever, the nanowire is initially compressed along the wire length and the cantilever is also deflected. The deflection of the cantilever, symbolically presented in Fig. 1(a) as $\Delta \theta$, is detected by the TEM system. With a calibrated force constant for the cantilever, the force applied to the nanowire can be determined. The compression of the nanowire, shown as $\Delta x$ in the diagram, can be determined in a sequence of TEM images as a function of an increasing load to the wire. Fig. 1(b) shows the typical TEM micrographs of a nanowire at different stages of the experiment: (i) the wire is touching the tip of the AFM cantilever; (ii) the wire is buckled under the applied force; and (iii) the wire restores to its original shape when it is withdrawn from the AFM cantilever, indicating the original deformation to be elastic. Fig. 1(c) displays a typical curve of force versus "apparent" nanowire displacement $(\Delta x) . \Delta x$ comprises both the nanowire compression and the indentation which the tip of the nanowire made on the surface of the cantilever. So, $\Delta x$ itself cannot be used reliably in the direct determination of the Young's modulus. However, two different elastic deformations can be clearly identified. The changeover point thus determined is the critical force of Euler buckling. The spring constant of the AFM cantilever was $0.3433 \mathrm{~N} / \mathrm{m}$, as calibrated by the thermal tune method well-established for AFM systems. ${ }^{16}$ Taking consideration of the experimental boundary condition and nanowire geometry, the theoretical model of a slender cylindrical beam with one end fixed and the other end pinned would be appropriate for the determination of the Young's modulus within the Euler buckling theory, in which the critical force of Euler buckling can be written as

$$
F_{c r}=\frac{\pi^{2} E I}{(0.7 l)^{2}},
$$

where $l$ is the length of the beam, $E$ is the Young's modulus, and $I$ is the area moment of inertia about the longitudinal axis of the beam determined by the nanowire geometry. The product $E I$ is known as the flexural rigidity. Experimental observations of the wire deformations suggest that the wires behave in a manner consistent with one end of the wire being fixed and the other pinned. A deviation from such a model would of course give rise to the effective factor of length being different from that of $0.7 l$, which would affect the value of $E$ being determined though Equation (1) and would introduce a systematic error to all experimental results of $E$. A single calibrated AFM cantilever was used for the present study, and the measurements of nanowires with the same size had been repeated five times to give an estimate of measurement errors.

Following the Euler-Bernoulli equation, ${ }^{7,11,12}$ the relationship of the resonance frequency $f$ of the transverse vibration of the wire and Young's modulus $E$ is as follows:

$$
f=\frac{\lambda_{j}^{2} d}{8 \pi l^{2}} \sqrt{\frac{E}{\rho}},
$$

where $\lambda_{j}$ is a constant for the $j_{\text {th }}$ harmonic, being 1.875 for the fundamental mode; $d$ is the diameter of the nanowire; and $\rho$ is the wire mass density. The density of a particular nanowire can thus be determined from Eq. (2).

For a typical Pt composite nanowire, which is $85 \mathrm{~nm}$ in diameter and $4.7 \mu \mathrm{m}$ in length, the average mass density thus determined is $12( \pm 1) \times 10^{3} \mathrm{~kg} / \mathrm{m}^{3}$, and the corresponding Young's modulus is about $2.0 \times 10^{2} \mathrm{GPa}$. The aspect ratio of the nanowire $l / d=55$ can be regarded as a slender nanowire, ${ }^{17}$ and the nanowire with the diameter of $85 \mathrm{~nm}$ is larger enough with weaker surface effects, ${ }^{18}$ for which the Euler 
buckling formula would be applicable. It is known that the FEB-CVD Pt consists of a significant amount of $\mathrm{C}$ and a very small amount of oxygen, and the chemical composition of the alloy can be written as $\operatorname{PtC}_{x}$ (where $x$ is between 4 and 8 and is dependent on the deposition conditions). ${ }^{19,20}$ Consequently, the mass density of FEB-CVD Pt nanowires is in the range of $(6-14) \times 10^{3} \mathrm{~kg} / \mathrm{m}^{3}$, while the density of the FEB-CVD Pt nanowire clearly lies within this range containing about $49 \%$ Pt. We note that the error in the length of the nanowire would be the major source for the subsequent error in the mass density as Eq. (2) was applied. However, the result we obtained was in general agreement with the available literature.

For electrically driven nanowires, static charges $\mathrm{Q}_{0}$, which can be compensated by a constant bias $\mathrm{V}_{0}$, are induced to balance the difference of the surface work functions between the nanowire and opposite $\mathrm{W}$ electrode. The induced charges can be described by $\alpha V_{d} \cos 2 \pi f t$, which is determined by the applied voltage and is time-dependent, where $\alpha$ is a geometrical factor, and $V_{\mathrm{d}}$ is the amplitude of the applied voltage. The amount of induced charges on the nanowire is $\mathrm{Q}=\alpha\left(V_{0}+V_{d} \cos 2 \pi f t\right)$. Meanwhile, the influence of the electron beam has been examined under the following conditions: an electron beam accelerating voltage of $200 \mathrm{kV}$, a magnification of X3000, and a corresponding beam current density of $32.5 \mathrm{pA} / \mathrm{cm}^{2}$; this will induce an accumulational charge $Q_{a}$, which is proportional to the current density on the nanowire. So, the final charge on the nanowire by considering all the effects is $\mathrm{Q}=\alpha\left(V_{0}+V_{d} \cos 2 \pi f t\right)+Q_{a}$. Therefore, the electrostatic force on the nanowire $F_{e}$ can be described as

$$
\mathrm{F}_{e}=\beta\left[\alpha\left(V_{0}+V_{d} \cos 2 \pi f t\right)+Q_{a}\right]\left(V_{0}+V_{d} \cos 2 \pi f t\right),
$$

where $\beta$ is the proportionality constant. ${ }^{7,8,11}$ Thus, the force exerted on the nanowire is expected to vary with the applied electric field strength, and basic vibration information can be derived accordingly. Alternatively, a similar effect can be observed by changing the inter-electrode distance of the two tips at a fixed excitation frequency and applied voltage. Since the exerted electro-static force is inversely proportional to the inter-distance of the electrodes squared, the amplitude of resonant vibration should decrease with the increasing inter-distance of the two tips.

In order to electrically drive the wire vibration, the sample was mounted on a second home-built TEM sample holder ${ }^{21,22}$ so that another $\mathrm{W}$ tip could be positioned close to the nanowire as shown in the inset of Fig. 2 (a) by a piezoelectrically driven mechanism. An a.c. voltage was applied to the two W tips. As the frequency of the applied voltage was increased, a transverse vibration of the nanowire was observed. Selected images, showing several stages of the vibration processes of a typical nanowire $6.6 \mu \mathrm{m}$ in length, $73 \mathrm{~nm}$ in diameter, and $1 \mu \mathrm{m}$ away from the fixed W tip, are presented in Fig. 2 (b). We note that the TEM imaging avoids the possible artefact in the observation of the vibrational modes which may exist in the raster scanning associated with SEM. ${ }^{9}$ The resonance amplitudes of the vibration and the first and the second harmonic vibrational modes were observed at different driving frequencies. We observed that it was easier to drive the second harmonic
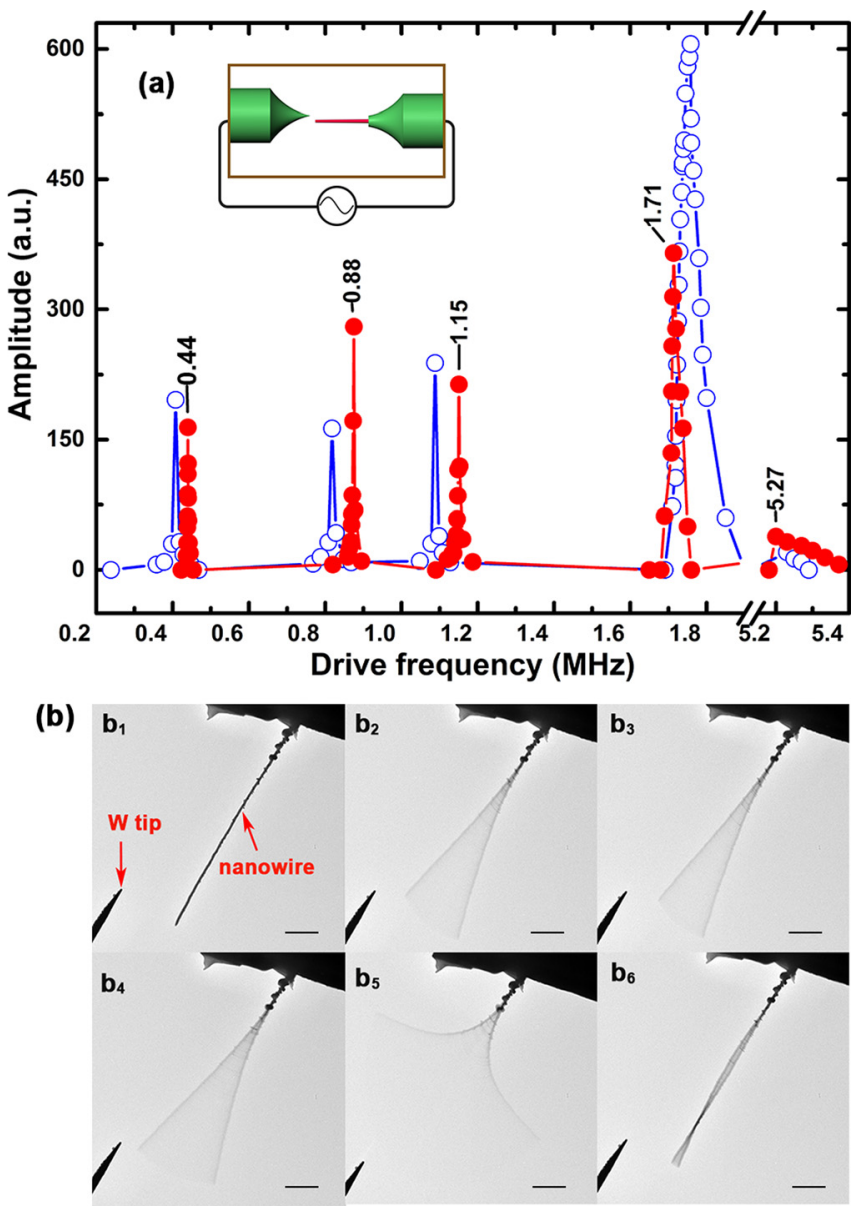

FIG. 2. Resonance of the FEB-CVD Pt composite nanowire: (a) the amplitude-frequency response curves with upward (solid red) and downward (hollow blue) frequency sweeping, respectively. The inset shows the schematic diagram of the experimental setup. (b) Observed vibrational modes of the nanowire: $\left(b_{1}\right)$ the stationary nanowire, $\left(b_{2}-b_{5}\right)$ the observed fundamental vibration mode of the nanowire at a drive frequency of $0.44 \mathrm{MHz}$, $0.88 \mathrm{MHz}, 1.15 \mathrm{MHz}$, and $1.71 \mathrm{MHz}$, and $\left(\mathrm{b}_{6}\right)$ second vibrational mode of the nanowire at a drive frequency of $5.2 \mathrm{MHz}$. The scale bar is $1 \mu \mathrm{m}$.

vibration for nanowires of a higher aspect ratio $l / d$, where $l$ and $d$ are the length and diameter of the nanowire, respectively. That is, the excitation signal frequency required to induce the high order resonance vibration for a slender nanowire is lower than that required for a thicker nanowire. However, the third and higher harmonic vibrations had not been observed even for nanowires with as a high aspect ratio as 110 , which may due to the fact that the frequency of the excitation signal was not high enough in our experiment. ${ }^{9}$ As shown in Fig. 2(a), the driving frequency values of $0.44 \mathrm{MHz}\left(\frac{f_{0}}{2}\right), 0.88 \mathrm{MHz}\left(f_{0}\right)$, and $1.71 \mathrm{MHz}\left(2 f_{0}\right)$ were observed to excite the fundamental mode of vibration of the nanowire. This multiple frequency resonance can be ascribed to the parametric resonance, ${ }^{23}$ which occurs close to the driving frequency $\omega=\frac{2 \omega_{0}}{n}(\mathrm{n}=1,2,3,4$, $5 \ldots$..., where $\omega_{0}=2 \pi f_{0}$ is the natural angular frequency of the wire. ${ }^{24} \mathrm{~A}$ force between the tungsten tip and the nanowire was applied, which has a component that is perpendicular to the nanowire length direction, so here the vibration modes are dominated by the forced excitation ${ }^{24}$ with the co-existence of the parametric vibration. ${ }^{23-25}$ For the particular nanowire concerned, the second harmonic vibration frequency $\nu_{2}$ was attributed to that at $(5.27 \pm 0.11) \mathrm{MHz}$, and at the fundamental 
vibration frequency $\nu_{1}(0.88 \pm 0.02) \mathrm{MHz}$, the value for $\nu_{2} / \nu_{1}$ is $(6.0 \pm 0.3)$. Within the experimental error, this value agrees with the theoretical ratio for a uniform cantilevered beam, which is 6.2, and suggests the vibrational modes' assignment to be selfconsistent. The driving frequency for the fundamental resonance frequency at $1.15 \mathrm{MHz}$ was unassigned, which may be caused by microstructural and chemical compositional anisotropy ${ }^{9}$ along the width of the examined nanowire, as can be evident by previously reported article $^{26}$ and further by the nanowire diameter related Young's modulus to be discussed later. A hysteresis was also observed (Fig 2(a)) with respect to the drive frequency sweep direction, highlighting the parametric nature of the driven oscillation.

In our experiment, the influence of the off-axis interelectrode distance between the nanowire situated $\mathrm{W}$ tip and the other W tip was examined, and Fig. 3(a) shows the resulting experimental curve. As expected, with the decreasing inter-electrode distance, the vibration amplitude increased while the resonance frequency is unchanged.

For the FEB-CVD nanowire, the mass density and the microstructures might vary with the growth conditions and geometry. ${ }^{11,12}$ From Fig. 3(b), it can be seen that with the increasing diameter of the nanowire, the Young's modulus
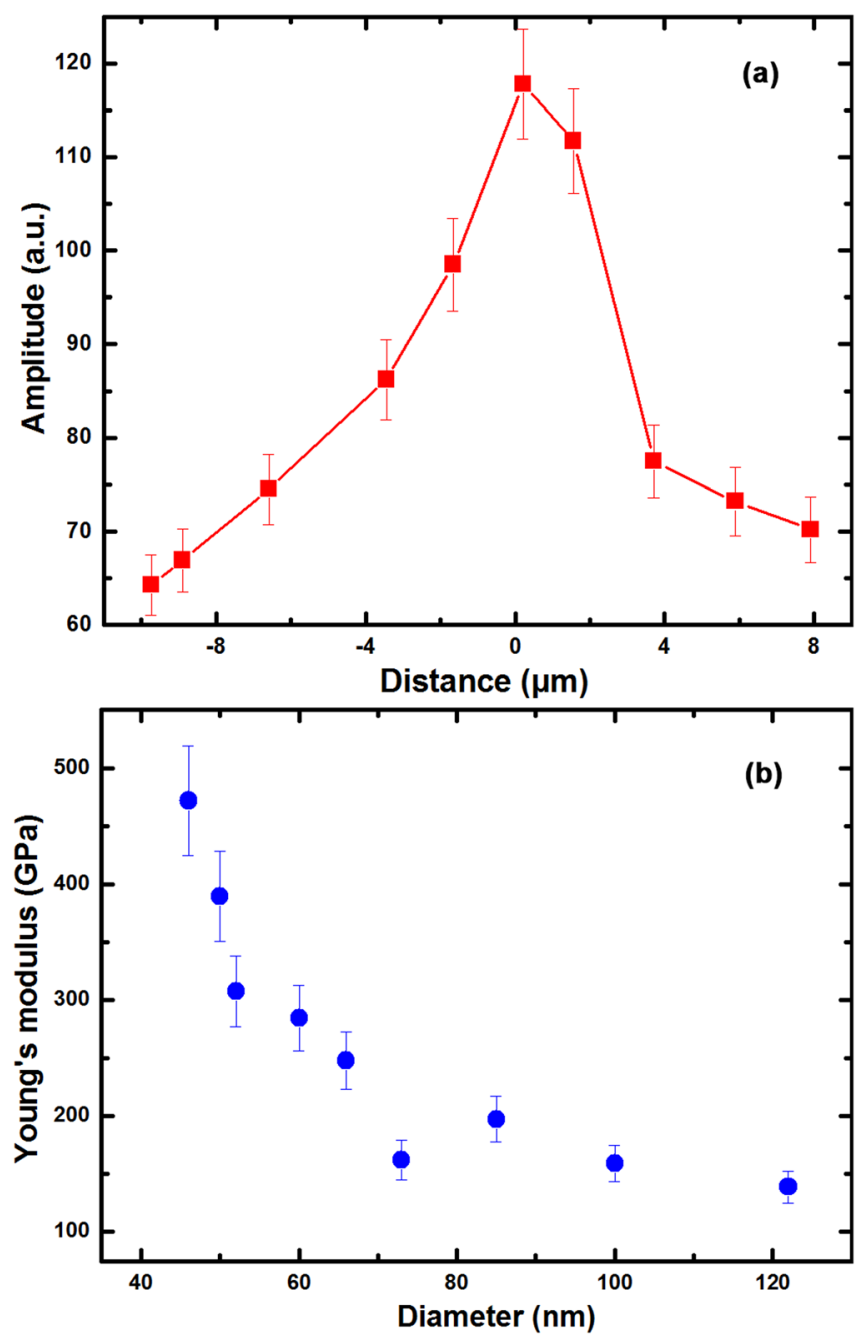

FIG. 3. (a) The dependence of the vibration amplitude and the distance between the two W tips and (b) the Young's modulus as a function of the diameter of the FEB-CVD grown Pt composite nanowires. decreases in the examined range and approaches a value about $160 \mathrm{GPa}$, which is comparearble with $168 \mathrm{GPa}$ of bulk $\mathrm{Pt}^{27,28}$ and $120 \mathrm{GPa}$ of the carbon pillar fabricated by FIB. ${ }^{13}$ This is similar to the trends previously reported. ${ }^{24,29}$ The reason for the significant higher values of Young's modulus of nanowires with smaller diameters is not yet entirely clear, although the surface properties and the micro-structure may play important roles, and there exists a strong size effect at the nanoscale scale. ${ }^{30-33}$ It is known that in nanowires with tens of nanometer in diameter, the surface modulus ${ }^{34}$ (that is determined by the surface state) is introduced, and the wires can be much more elastic ${ }^{31}$ when compared with their bulk counterparts. With the decreasing diameter, surface effects are expected to be more significant.

The observation of the unassigned resonance peak appeared to be consistent with the thermal behaviors, for instance, the bending of the wires under thermal annealing, ${ }^{26}$ which indicated the likely microstructural/chemical complications. A further experimental study is clearly required to identify the precise cause for its existence.

To examine the mass sensing ability of the nanowire systems, a preliminary demonstration was performed by measuring the resonance of a particular nanowire and then by adding an additional amount of mass on the top end of it and repeating the resonant measurement. The extra mass was grown using the FEB-CVD method. Control samples of two nanowires with nominally identical growth conditions showed negligible differences in their vibrational characteristics. Hence, nominally identical growth conditions were used to grow two nanowires with the second wire having an extra mass grown on the top end. Amplitude-frequency response curves were obtained experimentally, and it was found that the difference was significant. The SEM images of nanowires, $50 \mathrm{~nm}$ in diameter and $5.0 \mu \mathrm{m}$ in length, without and with additional mass are shown in Figs. 4(a) and 4(b), respectively. The corresponding amplitude and drive frequency response curves are shown in Fig. 4(c), and a significant fundamental resonant frequency shift was observed with the shift obviously larger than the estimated experimental error associated with the measurements. It is worth noting that the frequency shifts have nearly the same value for the

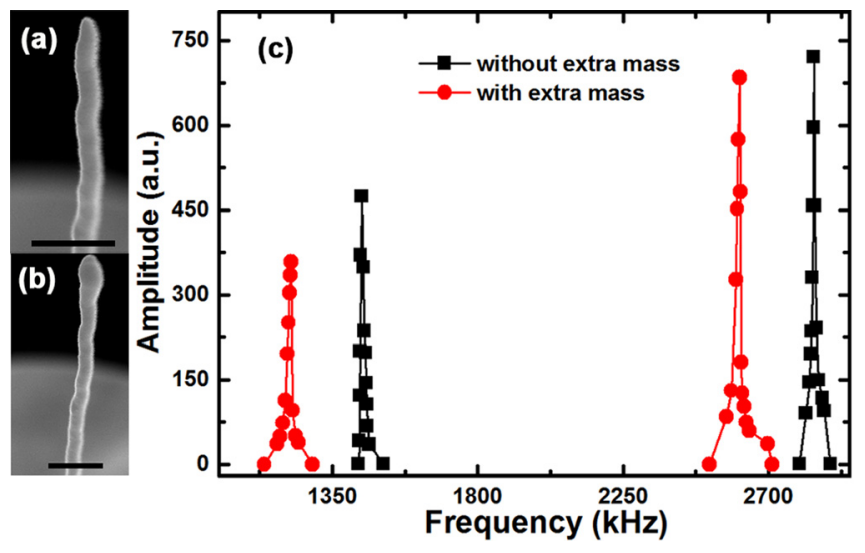

FIG. 4. Preliminary results demonstrating mass detection using the nanowire nano-balance: SEM images of (a) an as-deposited nanowire resonator and (b) of that with extra mass at the top end. (c) The amplitude-frequency response of the nano-balances. The scale bar is $200 \mathrm{~nm}$. 
fundamental frequency and the second harmonic frequency. The extra loaded mass increment $\Delta m$, estimated from the density and volume of the deposited material, is around $5.96 \times 10^{-18} \mathrm{~kg}$, and the mass responsivity $\Re$, defined as $\Re=\frac{\partial f}{\partial m_{e f f}}\left(m_{e f f}\right.$ is the effective vibratory mass $),{ }^{2,4,35}$ is estimated to be about $2.1 \times 10^{21} \mathrm{~Hz} / \mathrm{kg}$. A measurement bandwidth $\Delta \mathrm{f}$ of $1.0 \mathrm{kHz}$ yields a frequency noise floor, $\delta w_{0} / 2 \pi$ $\approx 80 \mathrm{~Hz}$, and the minimum detectable mass $\delta \mathrm{m}=\delta w_{0} / \Re$ $=3.8 \times 10^{-20} \mathrm{~kg}$. Thus, the minimum detectable mass is about 0.4 attogram (1attogram $=10^{-18} \mathrm{~g}$ ). ${ }^{4}$ If the small mass is attributed to a slight increase in the length of the wire, resulting in a small increase in the aspect ratio, for the FEBCVD Pt composite nano-resonator, the decrement in the resonant vibration frequency is calculated to be $253 \mathrm{kHz}$. This would mostly account for the large reduction in the resonance frequency (about $8.9 \%$ in frequency reduction), which is in good agreement with our experimental data of about $8.1 \%$ in frequency reduction. Moreover, the results are in agreement with the numerical calculation results from the energy of the beam. ${ }^{36}$ The detectable minimum mass, although it is not larger than that of the carbon tube with a similar experiment condition, ${ }^{2}$ is larger than nanomechanical resonator made with other materials and may be further improved with a decreased wire diameter.

In conclusion, we have fabricated "nano-balances" with a freestanding Pt composite nanowire on a $\mathrm{W}$ microprobe tip. A maximum Young's modulus of $(4.7 \pm 0.2) \times 10^{2} \mathrm{GPa}$ was estimated for the wires of the smallest diameter, and such a balance is capable of detecting small mass as low as 0.4 attogram with a sensitivity of $2.1 \times 10^{21} \mathrm{~Hz} / \mathrm{kg}$. Meanwhile, a straightforward experimental setup has been demonstrated to measure the Young's modulus value of a freestanding nanowire in situ in a TEM system. While the mass sensitivity is to be further improved, the nanowires produced by a fast and flexible fabrication method had been demonstrated to have a reasonably high mass sensitivity. We would expect that such a method of direct fabrication and integration of micro-balances on any given conductive surfaces can produce devices which may find their way in advanced electromechanical applications.

This work was supported by the National Natural Science Foundation of China under Grants Nos. 11574368, 11574369, 11674387, 91323304, and XDB07020200 and the National Key Research and Development Program under Grant Nos. 2016YFA0200400 and 2016YFA0200803.
${ }^{1}$ K. L. Ekinci and M. L. Roukes, Rev. Sci. Instrum. 76, 061101 (2005).

${ }^{2}$ K. Jensen, K. Kim, and A. Zettl, Nat. Nanotechnol. 3, 533 (2008).

${ }^{3}$ J. Moser, J. Guttinger, A. Eichler, M. J. Esplandiu, D. E. Liu, M. I. Dykman, and A. Bachtold, Nat. Nanotechnol. 8, 493 (2013).

${ }^{4}$ K. L. Ekinci, X. M. H. Huang, and M. L. Roukes, Appl. Phys. Lett. 84, 4469 (2004)

${ }^{5}$ M. D. LaHaye, O. Buu, B. Camarota, and K. C. Schwab, Science 304, 74 (2004).

${ }^{6}$ H. B. Chan, V. A. Aksyuk, R. N. Kleiman, D. J. Bishop, and F. Capasso, Science 291, 1941 (2001).

${ }^{7}$ P. Poncharal, Z. L. Wang, D. Ugarte, and W. A. de Heer, Science 283, 1513 (1999)

${ }^{8}$ Z. L. Wang, R. P. Gao, P. poncharal, W. A. de Heer, Z. R. Dai, and Z. W. Pan, Mater. Sci. Eng. C 16, 3 (2001).

${ }^{9}$ D. A. Dikin, X. Chen, W. Ding, G. Wagner, and R. S. Ruoff, J. Appl. Phys. 93, 226 (2003).

${ }^{10}$ J. Igaki, K. Kanda, Y. Haruyama, M. Ishida, Y. Ochiai, J. Fujita, T. Kaito, and S. Matsui, Microelectron. Eng. 83, 1225 (2006).

${ }^{11}$ Z. L. Wang, Z. Dai, R. Gao, and J. Gole, J. Electron Microsc. 51, s79 (2002).

${ }^{12}$ M. Ishida, J. Fujita, and Y. Ochiai, J. Vac. Sci. Technol. B 20, 2784 (2002).

${ }^{13}$ K. Nonaka, K. Tamaru, M. Nagase, H. Yamaguchi, S. Warisawa, and S. Ishihara, Jpn. J. Appl. Phys., Part 2 47, 5116 (2008).

${ }^{14}$ L. Ji, S. Young, T. Fang, and C. Liu, Appl. Phys. Lett. 90, 033109 (2007).

${ }^{15}$ C. Hsin, W. Mai, Y. Gu, Y. Gao, C. Huang, Y. Liu, L. Chen, and Z. L. Wang, Adv. Mater. 20, 3919 (2008).

${ }^{16}$ J. L. Hutter and J. Bechhoefer, Rev. Sci. Instrum. 64, 1868 (1993).

${ }^{17}$ G. Wang and X. Feng, J. Phys. D: Appl. Phys. 42, 155411 (2009).

${ }^{18}$ H. S. Park, Comput. Mater. Sci. 51, 396 (2012).

${ }^{19}$ H. Plank, J. H. Noh, J. D. Fowlkes, K. Lester, B. B. Lewis, and P. D. Rack, ACS Appl. Mater. Interfaces 6, 1018 (2014).

${ }^{20}$ S. Frabboni, G. C. Gazzadi, L. Felisari, and A. Spessot, Appl. Phys. Lett. 88, 213116 (2006).

${ }^{21}$ Z. Xu, X. D. Bai, and E. G. Wang, Appl. Phys. Lett. 88, 133107 (2006).

${ }^{22}$ Z. Xu, X. D. Bai, E. G. Wang, and Z. L. Wang, Appl. Phys. Lett. 87, 163106 (2005).

${ }^{23}$ M. F. Yu, G. J. Wagner, R. S. Ruoff, and M. J. Dyer, Phys. Rev. B 66, 073406 (2002).

${ }^{24}$ C. Q. Chen, Y. Shi, Y. S. Zhang, J. Zhu, and Y. J. Yan, Phys. Rev. Lett. 96, 075505 (2006).

${ }^{25}$ X. Y. Kong, Y. Ding, R. Yang, and Z. L. Wang, Science 303, 1348 (2004).

${ }^{26}$ A. Cui, W. Li, T. H. Shen, Y. Yao, J. C. Fenton, Y. Peng, Z. Liu, J. Zhang, and C. Gu, Sci. Rep. 3, 2429 (2013).

${ }^{27}$ M. C. Salvadori, I. G. Brown, A. R. Vaz, L. L. Melo, and M. Cattani, Phys. Rev. B 67, 153404 (2003).

${ }^{28}$ Z. Ma, H. Zhao, and L. Ren, Meas. Sci. Technol. 27, 067001 (2016).

${ }^{29}$ G. Y. Jing, H. L. Duan, X. M. Sun, Z. S. Zhang, J. Xu, Y. D. Li, J. X. Wang, and D. P. Yu, Phys. Rev. B 73, 235409 (2006).

${ }^{30}$ D. D. D. Ma, C. S. Lee, F. C. K. Au, S. Y. Tong, and S. T. Lee, Science 299, 1874 (2003).

${ }^{31}$ J. He and C. M. Lilley, Nano Lett. 8, 1798 (2008).

${ }^{32}$ P. M. Ajayan and T. W. Ebbesen, Rep. Prog. Phys. 60, 1025 (1997).

${ }^{33} \mathrm{C}$. Li and T. Chou, Compos. Sci. Technol. 63, 1517 (2003).

${ }^{34}$ G. Wang and X. Feng, Appl. Phys. Lett. 94, 141913 (2009).

${ }^{35}$ T. Ono and M. Esashi, Rev. Sci. Instrum.76, 093107 (2005).

${ }^{36}$ M. Gürgöze, J. Sound Vib. 190, 149 (1996). 